\title{
An Efficient Method for Detecting Covered Face Scenarios in ATM Surveillance Camera
}

\author{
Tasriva Sikandar ${ }^{\mathrm{a}^{*}}$, W Nur Azhani W Samsudin ${ }^{\mathrm{b}}$ and Mohammad Fazle Rabbi ${ }^{\mathrm{c}}$, Kamarul Hawari Ghazali ${ }^{\mathrm{d}}$

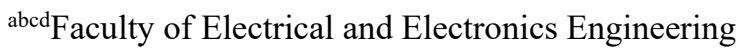 \\ abcdUniversiti Malaysia Pahang, 26600 Pekan, Pahang, Malaysia

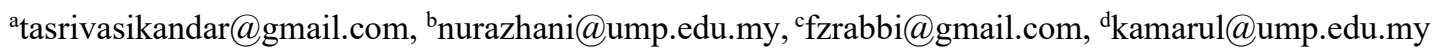

\begin{abstract}
Covering face with accessories such as mask, scarf and sunglass is a common criminal activity in Automated Teller Machine (ATM) robbery. Therefore, detection of covered face using ATM surveillance camera can be an effective solution to reduce robbery or crime. This paper presents a novel method to detect covered face from ATM surveillance camera images. Specifically, three facial features, i.e. skin color, elliptical face shape and facial Width-to-Height ratio $(f W H R)$, incorporated with geometrical property of ellipse have been employed to estimate the covered region. In addition, three parameters, i.e. facial area, $f W H R$ and covered area percentage, have been utilized for reliable classification. Experiment results demonstrate that the method can detect full covered, uncovered and partially covered faces at a correct detection rate of $98.3 \%, 93.3 \%$ and $97.78 \%$ respectively. The overall correct detection rate is $96.48 \%$, which is found to be better than previous studies. Also, the proposed method can handle faces covered with few new face hiding objects such as hijab, niqab and robber's ski mask. Furthermore, processing time of the proposed algorithm is significantly improved while compared to the existing methods. The detection time varies between $31 \mathrm{~ms}$ to $67 \mathrm{~ms}$ which is equivalent to $15-32$ frames per second.
\end{abstract}

Keywords: Auto teller machine, ATM crime, covered face, facial Width-to-Height Ratio, surveillance camera, ellipse. 


\section{Introduction}

According to recent reports criminals usually cover their faces with accessories such as masks, scarves and sunglasses during ATM crimes [1-2]. With the advances in image processing technology, surveillance camera can be used to detect covered face and, to give warning alarm immediately during any ATM crime. Although there are numerous covered face detection algorithms available in current literature, the conventional state of the art algorithms may not work for ATM surveillance due to different environment (illumination), captured scene (camera view, camera-user distance) and face covering accessories. Therefore extensive research has been carried out concentrating specifically on application of covered face detection methods in ATM surveillance [3-10].

Although large number of studies has been found on application of covered face detection methods in ATM surveillance, most of the methods lacked to consider real ATM surveillance captured scene (camera view, camerauser distance) and face covering accessories. A detail review of the previous studies on image processing integrated ATM video surveillance has been discussed in our systematic review in [11]. Previous methods were found employing variety of trained detectors, classifiers, complex functions and highly iterative algorithms such as, Gabor wavelets, Principal Component Analysis (PCA), Nearest Neighbor (NN), Chi-square distance and ellipse fitting. In addition, previous methods were designed based on some facial features to detect covered face. For example, single facial feature, i.e. facial component (eye, nose, mouth) was considered by [3-7]. Other studies [8-10] have been found combining facial features such as skin color, elliptic face shape and omega $(\Omega)$ headshoulder shape to form a stronger feature. Note that, another anthropometric but unique features such as, facial width and height has not been considered yet by the previous studies. Moreover, most of the previous studies were found detecting only few common face covering accessories such as hat $[4,10]$, sunglass $[3,6-8,10]$, mask $[3$, $8,10]$ and scarf [6]. Those studies did not consider commonly used attire (hijab and niqab) and robber's ski mask. Besides, previous studies were found experimenting with either videos recorded by image acquisition apparatus or images collected from popular face databases. More importantly, most of the studies did not give attention to real ATM surveillance camera arrangement (e.g. camera type, camera-user distance and captured view) during data collection. Usually, hidden front camera installed in an ATM machine captures close view with details of user's face image from approximately 2 feet $(61 \mathrm{~cm})$ distance between the user and ATM machine. Subsequently, the front camera is useful for analyzing and identifying the facial features of a potential criminal with covered faces in ATM proximity [12-14]. Therefore, it is necessary to consider real life camera arrangement to develop a practical method for ATM surveillance application.

Previous studies employed trained, complex and highly iterative methods incorporate with variety of facial features for better detection. But most of the studies experimented with limited covered face scenarios and real ATM camera setup during data collection. This article proposes a novel covered face detection method based on mathematical formulation of Facial Width-to-Height Ratio ( $f W H R$ ) incorporate with geometrical property of ellipse for ATM surveillance application. In addition, the proposed method has been tested by images recorded with standard ATM front camera setup and, with a variety of possible face covering accessories such as hijab, niqab and robber's ski mask.

\section{Background of fWHR and Unique Ellipse property}


The concept of $f W H R$ theory originates from visual arts known as "Rule of thirds". Specifically, this guideline of image composition asserts that a visual image of human face (e.g. photographs, portraits, film and design) should be divided into equally spaced horizontal thirds and vertical thirds [15]. The study by [16] depicts human face image's horizontal thirds (Fig. 1a) and other studies [17-18] illustrate measure of $f W H R$ as shown in Fig. 1b and Eq. (1).

$$
\begin{gathered}
f W H R=\frac{\text { Bizygomatic width }}{\text { Glabella to Subnasale height }} \\
f W H R=\frac{\text { Facial width }}{1 / 3 \text { of facial height }}
\end{gathered}
$$

The Eq. (1) is written in a general form as Eq. (2) where $1.44 \leq f W H R \leq 2.12$ for people of different races and gender [17-18]. Facial width and height will be represented by $W_{t}$ and $H_{t}$ respectively. The $f W H R$ concept has been applied in applications such as medical science (e.g. plastic surgery, physiological trait) and sports science (e.g. behavioral disposition) studies and demographic research [17-18]. However, most of the previous studies and applications are based on statistical data such as population survey research. Furthermore, facial region is usually considered as elliptical shape. From the geometrical property of an ellipse it can be shown that, the Euclidean distance between the top most and bottom most as well as, the left most and right most points of an ellipse are unique and can be used as facial feature (Fig. 1c). In this study, mathematical formulation of $f W H R$ concept and geometrical property of ellipse will be used to estimate different nature of partially covered face area.

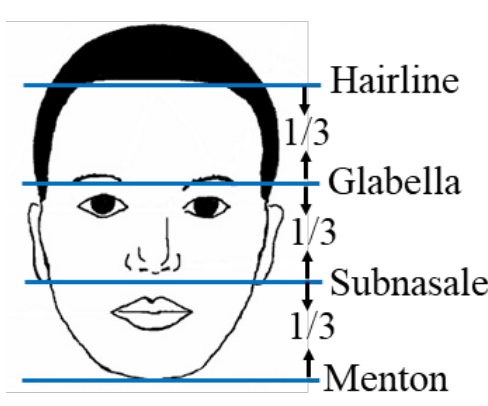

(a)

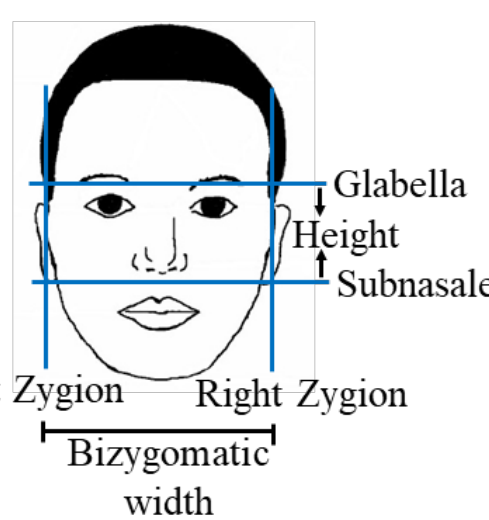

(b)

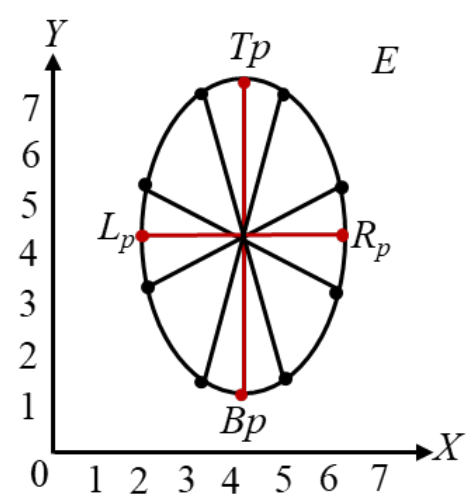

(c)

Fig. 1. (a) Facial horizontal thirds (b) measure used for facial Width-to-Height Ratio ( $f W H R$ ). (c) Unique ellipse property of Euclidean distance between the top most point $T_{p}$ and bottom most point $B_{p}$ as well as, the left most point $L_{p}$ and right most point $R_{p}$.

\section{Proposed Method}


Fig. 2 illustrates an overview of the proposed method and detail description of the proposed method is given below.

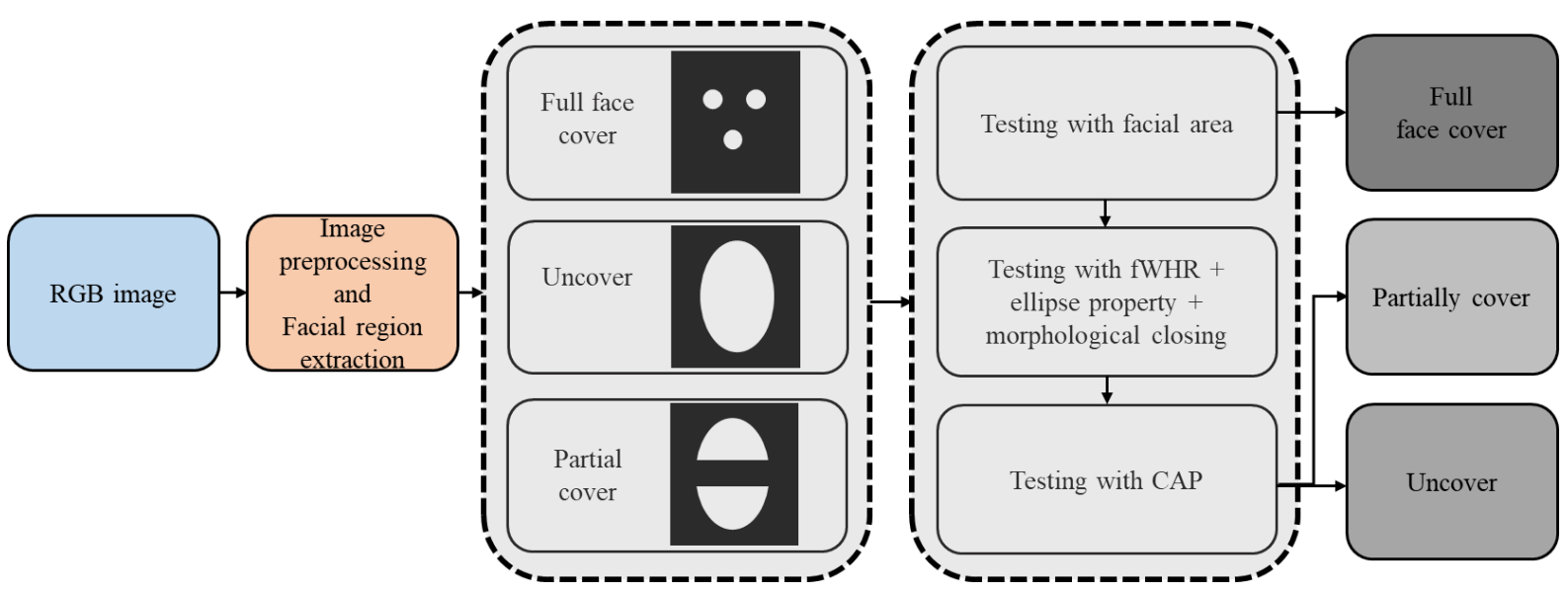

Fig. 2. An overview of the proposed method.

\subsection{Image Preprocessing and Facial Region Extraction}

The input RGB images are size normalized by down sampling the original size of input image to $250 \times 250$ pixel using MATLAB ${ }^{\odot}$ function imresize(). Facial region is detected using skin color information in $\mathrm{YCbCr}$ color space. As influence of illumination causes noise during image conversion process, the input RGB image is illumination compensated by using Gray world algorithm [19] prior to converting into YCbCr. Skin pixel classification is performed applying thresholding approach on pixel values. Threshold value range for $\mathrm{Cb}$ and $\mathrm{Cr}$ is defined as $\mathrm{Cb}=\left[\begin{array}{ll}77 & 127\end{array}\right]$ and $\mathrm{Cr}=\left[\begin{array}{ll}133 & 173\end{array}\right]$. Note that, $\mathrm{YCbCr}$ color space incorporate with Gray world algorithm and the $\mathrm{YCbCr}$ threshold value range $\mathrm{Cb}=\left[\begin{array}{ll}77 & 127\end{array}\right]$ and $\mathrm{Cr}=\left[\begin{array}{ll}133 & 173\end{array}\right]$ has been found to be an effective approach against illumination and different skin tone [20]. The facial region is extracted in the form of a binary image with skin color pixel as 1 (white). Then, the binary face image is further processed to detect different nature of covered faces. Fig. 3 illustrates the steps of image preprocessing and facial region extraction.

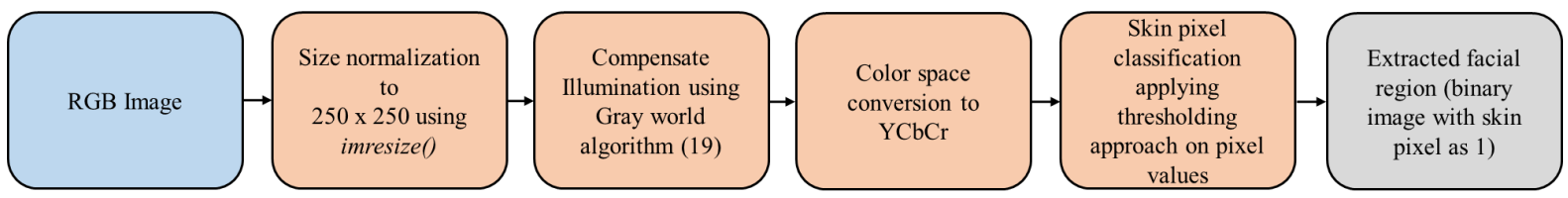

Fig. 3. Image preprocessing and facial region extraction step.

\subsection{Covered/Uncovered Face Detection}

In the proposed algorithm, the area of facial region $\left(A_{f r}\right)$ is calculated with the total number of 1 (white) pixel in the binary face image. The value of $A_{f r}$ is checked to compare with facial area threshold value $\left(t_{1}\right)$. If $A_{f r}$ is less than a certain threshold value, the face is categorized as "full face cover". Here, the threshold value is considered as $t_{1}=3300$. This threshold value is justified, in our experiments, to be optimal among different types of full face covering accessories (given in Appendix A). If $A_{f r}>t_{1}$, then the binary face image is further checked to find out whether the face is partially covered or uncovered. 
For partially covered and uncovered face checking, the first step is to remove noise from the binary face image by deleting the smallest objects to allow only the facial region to remain in the image. The method considers the facial region as elliptical shape similar to the previous studies. Then, the algorithm determines four points, i.e. top, bottom, left and right most points from the facial region in noise free binary face image. The algorithm considers Euclidean distance between top and bottom most points $\left(H_{t}\right)$ and left and right most point $\left(W_{t}\right)$ as facial region's height and width respectively. Fig. 4 demonstrates the location of determined four points, $H_{t}$ and $W_{t}$ in different nature of covered face scenarios such as uncovered, upper middle covered, lower middle covered, middle covered and lower portion covered. The algorithm then calculates $f W H R$ according to Eq. (2). $f W H R$ of facial scenarios given in Fig. 4a-4d satisfies the value range $1.44 \leq f W H R \leq 2.12$, whereas $f W H R$ of facial scenario Fig. $4 \mathrm{e}-4 \mathrm{f}$ follows $f W H R \geq 2.12$ or $f W H R \leq 1.44$. Based on the $f W H R$ value behavior for different covered face scenarios, the algorithm estimates covered region of face by following one of the two procedures: $(i)$ Procedure 1 or (ii) Procedure 2. If the condition $1.44 \leq f W H R \leq 2.12$ is satisfied, Procedure 1 is followed. If $f W H R \geq 2.12$ or $f W H R \leq 1.44$ is satisfied the algorithm follows Procedure 2.

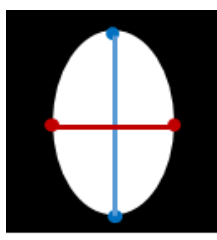

(a)

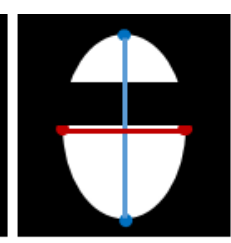

(b)

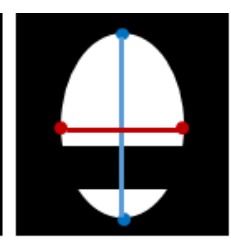

(c)

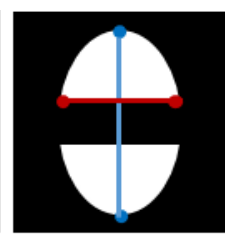

(d)

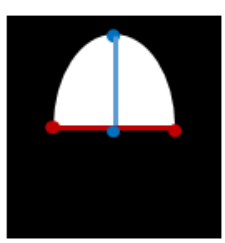

(e)

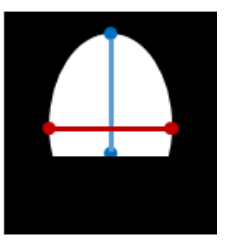

(f)

Fig. 4. Location of top, bottom, left and right most points in different facial scenario (a) uncovered (b) upper middle covered (c) lower middle covered (d) middle covered (e)-(f) lower portion covered. White area represents the skin area. Blue and red dots represent top-bottom most and left-right most points respectively. Blue line and red line represent $H_{t}$ and $W_{t}$, respectively.

Procedure 1- The algorithm applies morphological closing operation on noise free binary face image to fill in the gaps in facial region. The covered region is extracted by subtracting the noise free binary face image from morphologically closed binary image. Then noise is removed from covered region to eliminate the extra pixels in the image after morphological closing is introduced. The area of covered region $\left(A_{c r}\right)$ is calculated with the 1 (white) pixels in noise free covered region. The covered area percentage $\left(P_{c a}\right)$, is obtained using $P_{c a}=\frac{A_{c r}}{A_{c r}+A_{f r}} \times 100$.

Procedure 2- As shown in Fig 5a, $H_{t}$ and $W_{t}$ indicate the determined facial height and determined facial width, respectively in a lower portion covered facial scenario. As depicted in Fig. 5b we assume maximum facial region with $f W H R=2.12$, where $\operatorname{Max}_{H t}$ indicate the maximum facial height. We also assume minimum facial region with $f W H R=1.44$, where $\operatorname{Min}_{H t}$ indicate minimum facial height. The algorithm derives $\operatorname{Max}_{H t}$ and $\operatorname{Min}_{H t}$ as $\operatorname{Max}_{H t}=\left(3 \times W_{t}\right) / 2.21 \quad$ and $\operatorname{Min}_{H t}=\left(3 \times W_{t}\right) / 1.44$ from Eq. (2). 
The method calculates the maximum and the minimum height of covered regions, which are denoted as $\operatorname{MaxC}_{H t}$ and $\operatorname{MinC}_{H t}$, respectively. These heights are determined by $\operatorname{MaxC}_{H t}=\left|\operatorname{Max}_{H t}-H_{t}\right|$ and $\operatorname{MinC}_{H t}=\left|\operatorname{Min}_{H t}-H_{t}\right|$ as illustrated in Fig 5c. The algorithm derives the maximum and the minimum area of covered region using ellipse area formula. As shown in Fig. 5c the method considers a maximum estimated ellipse, $e_{\max }$ with $\operatorname{Max}_{H t}$ as semi major axis, and half of the facial width $\left(0.5 \times W_{t}\right)$ as semi-minor axis. Similarly, the method considers a minimum estimated ellipse, $e_{\min }$ with $\operatorname{MinC}_{H t}$ and half of the facial width $\left(0.5 \times W_{t}\right)$ as semimajor axis and semi-minor axis, respectively.

Therefore, the maximum and the minimum area of covered region, i.e. $M a x A_{c r}$ and $\operatorname{Min} A_{c r}$, can be derived from these two estimated ellipse $e_{\max }$ and $e_{\min }$ according to Eq. (3) and Eq. (4), respectively.

$$
\begin{aligned}
& \operatorname{MaxA}_{c r}=0.5 \times \text { Area of } e_{\max }=0.5 \times \pi \times\left(\operatorname{Max}_{H t} \times\left(0.5 \times W_{t}\right)\right) \\
& \text { MinA }_{c r}=0.5 \times \text { Area of } e_{\min }=0.5 \times \pi \times\left(\operatorname{Min}_{H t} \times\left(0.5 \times W_{t}\right)\right)
\end{aligned}
$$
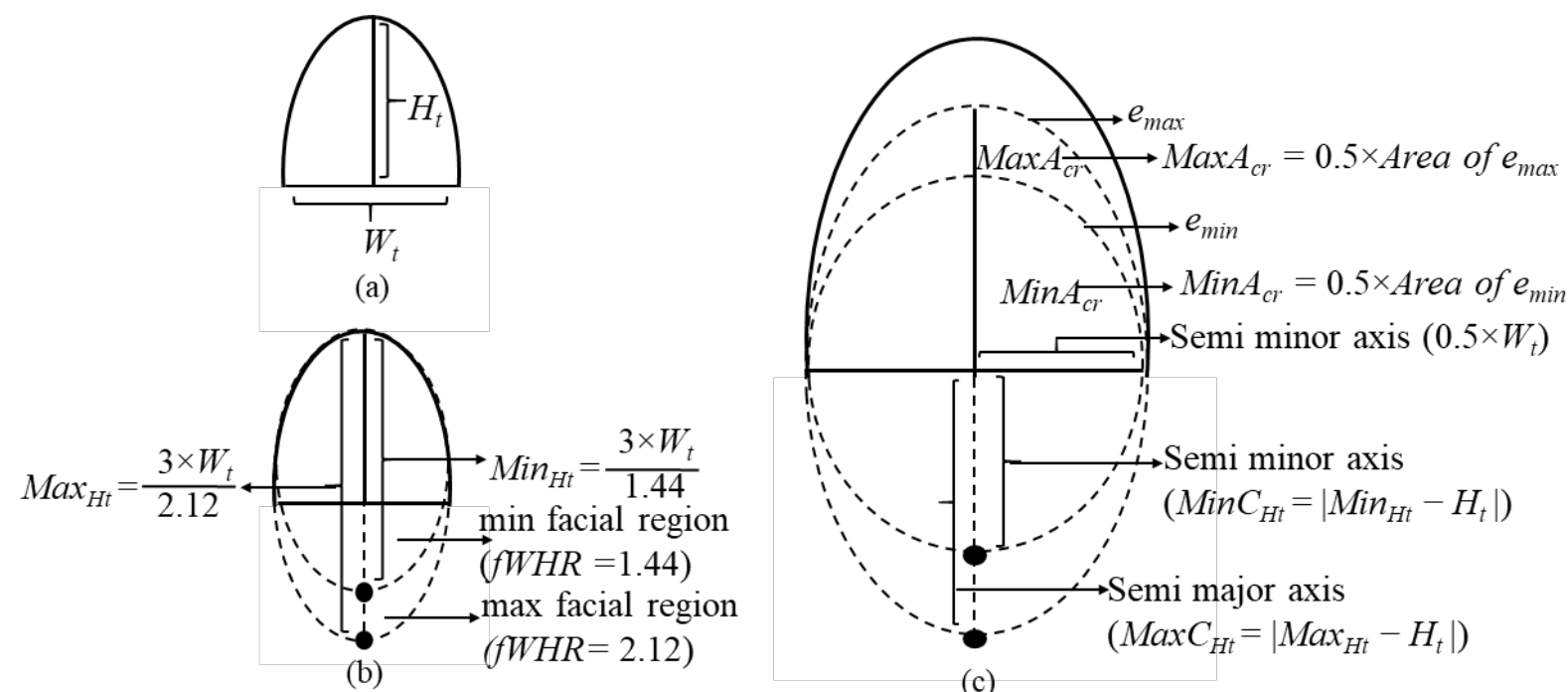

(c)

Fig. 5. Covered region area calculation procedure 2 (a) lower portion cover facial scenario with determined facial height and width (b) the maximum and the minimum facial region assumption (c) ellipse estimation for maximum and minimum covered region calculation. $H_{\mathrm{t}}$ and $W_{\mathrm{t}}$ are determined facial height and width, respectively. Max $x_{\mathrm{Ht}}$ and $\operatorname{Min}_{\mathrm{Ht}}$ are maximum and minimum facial height, respectively. $\operatorname{Max} C_{\mathrm{Ht}}$ and $\operatorname{Min} C_{\mathrm{Ht}}$ are maximum and minimum covered region height, respectively. $e_{\max }$ and $e_{\min }$ are maximum and minimum estimated ellipse, respectively. $\operatorname{Max} A_{\mathrm{cr}}$ and $\operatorname{Min} A_{\mathrm{cr}}$ are maximum and minimum covered region area, respectively.

The covered area percentage is determined according to Eq. (5) and equation (6) to provide the maximum and the minimum estimate of covered region for partially covered face.

$$
\begin{aligned}
& \operatorname{Max}_{c a}=\frac{\operatorname{Max} A_{c r}}{\operatorname{Max} A_{c r}+A_{f r}} \times 100 \\
& \operatorname{Min} P_{c a}=\frac{\operatorname{Min}_{c r}}{\operatorname{Min} A_{c r}+A_{f r}} \times 100
\end{aligned}
$$


The algorithm confirms the decision using thresholding-based classification approach. If the estimated covered area percentage is less than a threshold $t_{2}$, the face is considered as uncover. On the other hand, if the covered area percentage is greater than $t_{2}$ the face is considered as partially cover. The value of $t_{2}=10 \%$ was found to be optimal among different types of uncover face and partial face covering accessories (see Appendix B).

Table 1. Summary of dataset

\begin{tabular}{lclcc}
\hline Scenarios & No of subject & Face hiding accessories & No. of image & Total image \\
\hline Uncover & 30 & Bare (hijab, non-hijab) & 90 & 180 \\
\cline { 2 - 4 } & & $\begin{array}{l}\text { Normal glass (hijab, non- } \\
\text { hijab) }\end{array}$ & 90 \\
\hline Full & Robber's ski mask & 90 & 180 \\
\cline { 2 - 4 } $\begin{array}{l}\text { cover } \\
\text { Partially }\end{array}$ & 30 & Niqab & 90 & 180 \\
cover & 30 & Medical mask, Scarves & 90 \\
\hline
\end{tabular}

\section{Experiment Design and Dataset}

Although there are few well-known face image database existed and have been used in the previous studies conducting research on covered face detection, those database are not compatible with ATM environment. In addition, the face images database from ATM camera is not easy to access due to the security of bank [11].Therefore, in this study we opt to create our own dataset considering the indoor ATM environment in university premises. ATMs located in indoor premises usually capture images in two illumination conditions: (i) indoor illumination and (ii) indoor illumination with presences of sunlight from a side. In addition, description of ATM front camera distance and captured view has been found from the previous study [12-14]. Subsequently, to conduct an experiment in a practical scenario a dataset has been constructed considering real ATM's front camera set up (camera distance and captured view), indoor ATM's illumination conditions and possible face covering scenarios.

The camera is set on a tripod which is placed at a distance of 2 feet from the subject capturing 2 feet vertical view of the subject. The camera can capture a close view of face along with head of the subject with this set up. The subjects are instructed to sit on a chair which was placed in front of a wall white background. In addition, the subjects had to keep their back upright and face straight. Only frontal face of the subject has been captured. Since the proposed method down sample the images to $250 \times 250$ pixels, the method is independent to the camera resolution. The images are captured using a 5MP camera. We took the image sequences from thirty (30) students (fifteen (15) male and fifteen (15) female) of different races. For instance, Malay, Chinese and Indian with different complexion of skin has been considered in this study. The students participated in the study voluntarily and provided their consent prior to the experiment. The image sequences are taken in indoor environment with two illumination conditions (i) room illumination and (ii) room illumination in the presence of sunlight (window side). To have variations in sunlight illumination as well as color and type of face covering accessories, the images are taken in three sessions such as morning (8am-10am), mid-day (12pm to $2 \mathrm{pm})$ and afternoon (5pm-7pm). 
The face image dataset consists of three scenarios: uncover, full cover and partially cover (Table 1). Each scenarios includes two different face covering accessories such as (i) uncover includes bare face and normal glass face including hijab (vale covering head) and non-hijab face (ii) fully cover includes robber ski mask and niqab (ruband covering head and face except eyes), and (iii) partially cover includes medical mask, scarves and sunglass faces. The scenarios also includes hijab and non-hijab faces. The dataset contains a total of 540 image sequences, in which 180 image sequences are used for each scenario.

\section{Results and Performance Evaluation}

This section presents and describes result of experiment. All experiment results have been calculated on a desktop computer with an Intel(R) Core(TM)i5-2400, 3.10 GHz CPU, 8 GB RAM, Intel(R) HD graphics 2000 GPU. Our experiment was performed in Windows 10 and with MATLAB 2014a software. Some examples of detection results are shown in Fig. 6- Fig. 8. Faces exposing minimal skin area $(\leq 3300$ pixels $)$ are detected as "full face cover". Faces having covered area $\leq 10 \%$ are detected as "uncover", whereas faces wearing covered area $\geq 10 \%$ are detected as "partially cover".

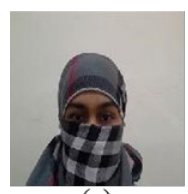

(a)

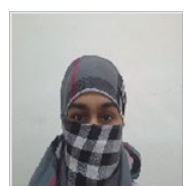

(b)

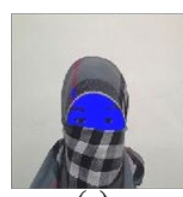

(c)

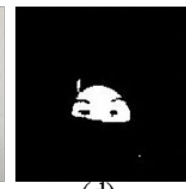

(d)

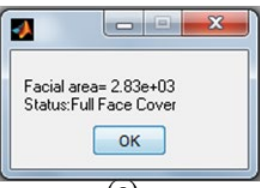

(e)

Fig. 6. Example of full cover face detection process and result (a) original image (b) illumination compensated image (c) skin pixel detected as blue in $\mathrm{YCbCr}$ color space (d) binary image with skin pixel 1 (white) (e) result.

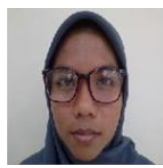

(a)

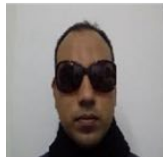

(a)

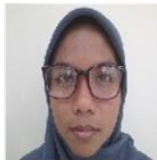

(b)

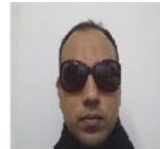

(b)

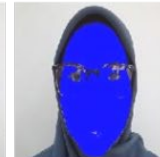

(c)

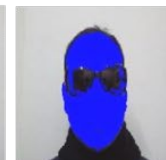

(c)

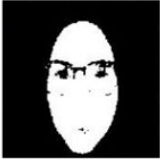

(d)

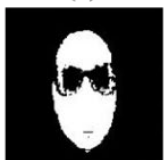

(d)
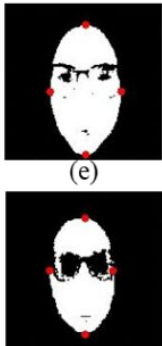

(e)
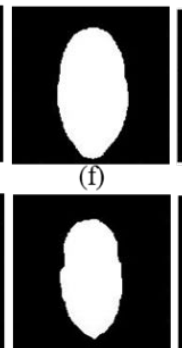

(f)

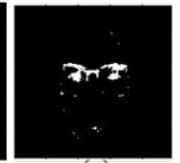

(g)

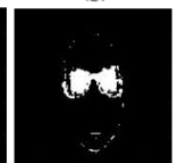

(g)

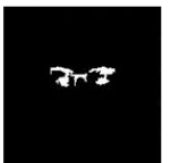

(h)

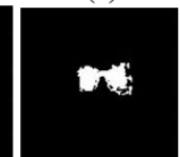

(h)

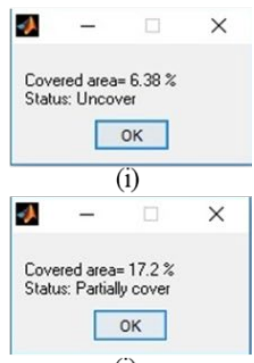

(i)

Fig. 7. Examples of uncover and partially cover face detection process following procedure 1 and result (a)original image (b) illumination compensated image (c) skin pixel detected as blue in YCbCr color space (d) binary image with skin pixel 1(white) (e) top, bottom, left and right most point determination (f) morphological closing (g) extracted covered area after image subtraction (h) covered area after noise removal (i) result.

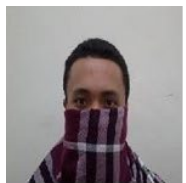

(a)

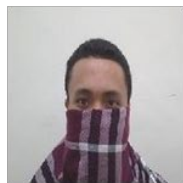

(b)

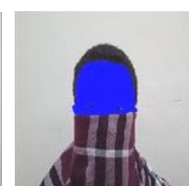

(c)

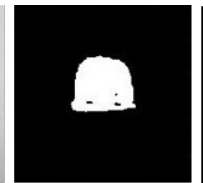

(d)

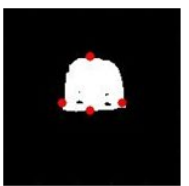

(e)

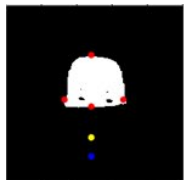

(f)

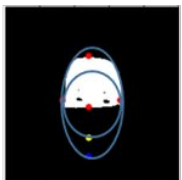

(g)

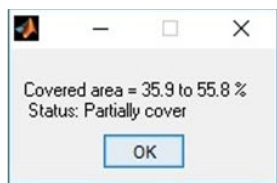

(h)

Fig. 8. Example of uncover and partially cover face detection process following procedure 2 and result (a) original image (b) illumination compensated image (c) skin pixel detected as blue in YCbCr color space (d) binary image 
with skin pixel 1(white) (e) top, bottom, left and right most point determination (f) maximum and minimum facial height calculation $(\mathrm{g})$ maximum-minimum covered region estimation using ellipse area formula $(\mathrm{h})$ result.

Performance of the proposed method is evaluated in terms of correct detection rate and detection time. Here, the correct detection rate is determined as

$$
\text { Correct Detection Rate }=\frac{N_{\text {correct }}}{N_{\text {correct }}+N_{\text {incorrect }}} \times 100
$$

Here $N_{\text {correct }}$ is number of correctly detecting a scenario and $N_{\text {incorrect }}$ is number of incorrectly detecting the scenario. Table 2 and Table 3 present the results of covered face detection and the correct detection rate performance of the proposed method, respectively. An average correct detection rate of $96.48 \%$ can be achieved using the proposed method. Specifically, 98.34\%, 93.33\% and 97.78\% correct detection rate were achieved for full face covered, uncovered, and partially covered faces respectively.

Table 2. Result of covered face detection.

\begin{tabular}{|c|c|c|c|c|c|}
\hline & & & \multicolumn{3}{|c|}{ Predictive class } \\
\hline & & & $\begin{array}{c}\text { Fully } \\
\text { covered }\end{array}$ & Uncovered & $\begin{array}{l}\text { Partially } \\
\text { covered }\end{array}$ \\
\hline \multirow{6}{*}{ 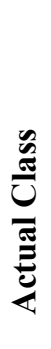 } & \multirow[t]{2}{*}{ Uncovered } & Bare (hijab, non-hijab) & 0 & 84 & 6 \\
\hline & & Normal glass (hijab, non-hijab) & 0 & 84 & 6 \\
\hline & \multirow{2}{*}{$\begin{array}{l}\text { Fully } \\
\text { covered }\end{array}$} & Robber's ski mask & 90 & 0 & 0 \\
\hline & & Niqab & 87 & 0 & 3 \\
\hline & \multirow{2}{*}{$\begin{array}{l}\text { Partially } \\
\text { covered }\end{array}$} & Medical mask, Scarves & 4 & 0 & 86 \\
\hline & & Sunglass (hijab,non-hijab) & 0 & 0 & 90 \\
\hline
\end{tabular}

Table 3. Correct detection rate performance of proposed method.

\begin{tabular}{|c|c|c|c|c|c|c|}
\hline Case & $\begin{array}{l}\text { Face covering } \\
\text { object }\end{array}$ & $\begin{array}{l}\text { No. of } \\
\text { image }\end{array}$ & $\begin{array}{l}\text { No. of } \\
\text { correct } \\
\text { detection }\end{array}$ & $\begin{array}{l}\text { No. of } \\
\text { wrong } \\
\text { detection }\end{array}$ & $\begin{array}{l}\text { Correct } \\
\text { detection } \\
\text { rate }(\%)\end{array}$ & $\begin{array}{l}\text { Avg. } \\
\text { Correct } \\
\text { detection } \\
\text { rate }(\%)\end{array}$ \\
\hline \multirow[t]{2}{*}{ Uncovered } & $\begin{array}{l}\text { Bare (hijab, non- } \\
\text { hijab) }\end{array}$ & 90 & 84 & 6 & 93.33 & \multirow[t]{2}{*}{93.33} \\
\hline & $\begin{array}{l}\text { Normal glass } \\
\text { (hijab, non-hijab) }\end{array}$ & 90 & 84 & 6 & 93.33 & \\
\hline \multirow{2}{*}{$\begin{array}{l}\text { Fully } \\
\text { Covered }\end{array}$} & Robber's ski mask & 90 & 90 & 0 & 100 & \multirow[t]{2}{*}{98.34} \\
\hline & Niqab & 90 & 87 & 3 & 96.67 & \\
\hline \multirow[t]{2}{*}{$\begin{array}{l}\text { Partially } \\
\text { covered }\end{array}$} & $\begin{array}{l}\text { Medical mask, } \\
\text { Scarves }\end{array}$ & 90 & 86 & 4 & 95.56 & \multirow[t]{2}{*}{97.78} \\
\hline & $\begin{array}{l}\text { Sunglass } \\
\text { (hijab,non-hijab) }\end{array}$ & 90 & 90 & 0 & 100 & \\
\hline
\end{tabular}

Table 4 presents detection time performance [21] of the proposed method for all face covering scenarios.

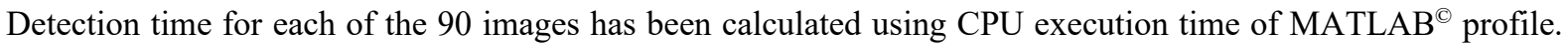
Then average detection time for each facial scenario has been calculated. The method can provide decision on full face cover scenarios (robber's ski mask, niqab) in less than $31 \mathrm{~ms}$ (Table 4) as only the first level of inspection is 
required for full face cover detection. Furthermore, the uncover (bare face, normal glass) and sunglass face detection require $61 \mathrm{~ms}$ and $67 \mathrm{~ms}$ respectively. The reason is, all three inspection level including $f W H R$ and morphological closing are executed for detection. On the other hand, to detect medical mask and scarves wearing face, the method executes all the three inspection level including $f W H R$ and ellipse property in $48 \mathrm{~ms}$. Overall, the proposed method can detect a face cover scenario in each image within $31 \mathrm{~ms}$ to $67 \mathrm{~ms}$ which is equivalent to a frame rate of approximately 15-32 fps.

Table 4. Detection time performance of the proposed method.

\begin{tabular}{|l|l|l|l|l|}
\hline Case & Face covering object & $\begin{array}{l}\text { Avg. detection } \\
\text { time (ms) }\end{array}$ & $\begin{array}{l}\text { Approx. } \\
\text { detection } \\
\text { time (ms) }\end{array}$ & $\begin{array}{l}\text { Approx. frame } \\
\text { rate (fps) }\end{array}$ \\
\hline \multirow{2}{*}{ Uncovered } & Bare (hijab, non-hijab) & 61 & $\approx 31-67$ & $\approx 15-32$ \\
\cline { 2 - 3 } & $\begin{array}{l}\text { Normal glass (hijab, non- } \\
\text { hijab) }\end{array}$ & 64 & \\
\hline $\begin{array}{l}\text { Fully } \\
\text { Covered }\end{array}$ & Robber's ski mask & 31 & \\
\cline { 2 - 3 } & Niqab & 33 & \\
\hline $\begin{array}{l}\text { Partially } \\
\text { covered }\end{array}$ & Medical mask, Scarves & 48 & \\
\cline { 2 - 3 } & Sunglass (hijab,non-hijab) & 67 & \\
\hline
\end{tabular}

\section{Discussion}

The performance of the present method was compared with that of the previous methods based on (i) data modeling (ii) respective correct detection rate, detection time and frame rate performance of the studies (iii) correct detection rate performance for the present study dataset and (iv) security camera standard.

Note that, although there are standard face databases, which are used by some of the previous studies [3,6], the images of these datasets are not captured according to ATM camera arrangement (camera distance and captured view). In addition, other studies $[4,5,7,8]$ that created their own dataset for ATM applications are mostly unavailable and have limitations such as camera arrangements, possible illumination and possible facial scenario. Therefore, we had to design experiment considering real ATM environment and model our own data set to show our results. Comparing with the previous studies [3-10], the experiment of the present study is designed considering real ATM's (located in indoor premises) environment such as illumination condition (indoor, indoor+sunlight), camera-user distance, captured view and possible face covering scenario. Also, the dataset of the present study includes some new and practical scenarios such as robber's ski mask, niqab and hijab faces.

Table 5. Contrast on respective detection rate, detection time and frame rate performance among the previous studies and the present study.

\begin{tabular}{llll}
\hline Methods & Detection rate (\%) & Detection time (ms) & Frames Processed (fps) \\
\hline kim G. et al $2010[8]$ & 86.7 & Not mentioned & Not mentioned \\
\hline Zhang T. et al 2018 $[10]$ & $\mathbf{9 8 . 5 6}$ & $\approx 83-166$ & $\approx 6-12$ \\
\hline Present method & 96.48 & $\approx \mathbf{3 1 - 6 7}$ & $\approx \mathbf{1 5 - 3 2}$ \\
\hline
\end{tabular}


Table 6. Comparison on correct detection rate performance between the method in [8] and the present method.

\begin{tabular}{lll}
\hline Cases & Methods & \\
\cline { 2 - 3 } & Kim G et al 2010 $[8]$ & Proposed method \\
\hline Bare Face (hijab, Non-hijab) & $75.56 \%$ & $\mathbf{9 3 . 3 3 \%}$ \\
\hline Normal glass (hijab, Non-hijab) & $73.33 \%$ & $\mathbf{9 3 . 3 3 \%}$ \\
\hline Robber Ski mask & Did not consider & $\mathbf{1 0 0 \%}$ \\
\hline Niqab & Did not consider & $\mathbf{9 6 . 6 7 \%}$ \\
\hline Medical mask, scarves & $87.78 \%$ & $\mathbf{9 5 . 5 6 \%}$ \\
\hline Sunglass & $91.11 \%$ & $\mathbf{1 0 0 \%}$ \\
\hline
\end{tabular}

Due to dissimilar experiment design, it is very difficult to make a fair comparison with other published investigations on detection of covered face scenario in ATM surveillance. However, the results can still be compared with some of the previous studies since they used some common features. For example, similar to our study, two recent $[8,10]$ are designed based on skin color and elliptic face shape feature. Comparison of correct detection rate, detection time and frame rate performance found in those studies and the present study has been shown in Table 5. The present study achieves better results in all manner than that of the study in [8]. Although the detection rate performance of the study in [10] outperforms the present study marginally, the detection time and frame rate performance of the present study is better than that of the study in [10]. In addition, comparing different face covering scenarios (Table 6) show that, our method performs better than the method by [8] for correctly detecting covered-uncovered facial scenarios. The method by [8] experiences difficulties in detecting hijab wearing faces, since hijab outline misguides to determine head-shoulder boundary and to apply ellipse fitting for face detection whereas using $f W H R$ our method can correctly detect hijab wearing faces. Moreover, the method by [8] did not consider very common scenarios e.g. robber's ski mask and niqab and thus experiences difficulties in detecting those scenarios, whereas the proposed method can detect those scenarios with high correct detection rate.

More importantly, the comparison implies that the covered face detection performance of the present study is appropriate for ATM surveillance system in terms of security camera standards. Conventional surveillance camera standard may require frame rate of as low as 1- $10 \mathrm{fps}$ [22] while $30 \mathrm{fps}$ has been considered as most of the security camera system so far [23]. Note that, if subject moves faster, higher frame rate is required for the identification process. However, if the subject stands still, the low frame rate would be enough to detect the covered face [24]. Therefore, it can be concluded that, the proposed method is suitable for ATM surveillance camera application. In addition to that, the proposed method can also be applied in other modality of capturing face image such as thermal face images [25-26].

Despite having rigorous effort, this study has few limitations. For example, in this study only indoor ATM environment has been considered. The outdoor light illumination has not been taken into consideration. Also, the background information of the images has also been considered as null. However, in a practical scenario, the background image may consist of any other user in a queue or other substance. Therefore, background information should be filtered properly to get reasonable output of the covered face detection method for ATM environment. These issues will be addressed in our future work. 


\section{Conclusions}

In this paper we have proposed a novel method for covered face detection of a variety of face covering scenarios in ATM environment. Three different face covering setups have been considered such as, uncover, partially cover and full cover. The proposed method utilizes three facial features, i.e. skin color, elliptical shape and $f W H R$ which is originated from visual arts. In this study, $f W H R$ has been proved to be a reliable tool to detect different kinds of covered faces. Specifically, geometrical property of ellipse and mathematical formulation of fWHR have been combined to develop a covered face detection method. Integrating with mathematically simple approach, the proposed method requires only four edge points to be extracted around the elliptic facial region. In addition to some common face covering accessories, the proposed method can detect faces with hijab, niqab and robber's ski mask. The correct detection rate of the proposed method is $96.48 \%$. Furthermore, the method can detect a variety of face covering scenarios in a significantly short period of $31 \mathrm{~ms}$ to $67 \mathrm{~ms}$ and can process approximately 15-32 frames per second. Being compatible with ATM front camera arrangement and frame rate requirement, the proposed method can be used in the application for covered face detection with ATM surveillance camera.

\section{Conflict of interest}

The authors declare no conflict of interest.

\section{Acknowledgement}

This research was supported by Universiti Malaysia Pahang (UMP) research grant scheme (No. RDU 1703256)

\section{References}

1. Graczyk M.: Masked men steal lobby ATMs from 5 Houston Marriott hotels. Daily Herald, Houston (14 Dec 2017). http://www.dailyherald.com/article/20171214/news/312149823. Accessed 17 Dec 2017.

2. Bromwich J. E.: A Smash-and-Grab Heist in Pennsylvania: Masked Men Steal an A.T.M.. The New York Times, Pennsylvania (1 Dec 2016). https://www.nytimes.com/ 2016/12/01/us/a-smash-and-grab-heist-inpennsylvania-masked-men-steal-an-atm.html. Accessed 20 Dec 2016.

3. Choi I. and Kim D.: Facial Fraud Discrimination Using Detection and Classification. In International Symposium on Visual Computing, pp. 199-208 (2010).

4. Yi M.: Abnormal Event Detection Method for ATM Video and Its Application. Adv. Res. Comput. Educ. Simul. Model. Berlin Heidelb., 186-192 (2011).

5. Jae Kyu S., Eum S., Gi H., Li G., Kim G. and Kim J.: Recognizability assessment of facial images for automated teller machine applications. Pattern Recognit., 45(5), 1899-1914 (2012).

6. Min R., Hadid A. and Jean-Luc D.: Efficient Detection of Occlusion prior to Robust Face Recognition. Sci. World J. (2014).

7. Ray S., Das S. and Anindya S.: An Intelligent Vision System for monitoring Security and Surveillance of ATM. In 2015 Annual IEEE India Conference (INDICON), IEEE, pp. 1-5 (2015).

8. Kim G., Suhr J. K., Jung H. G. and Jaihie K.: Face Occlusion Detection by using B-spline Active Contour and Skin Color Information. In Control Automation Robotics \& Vision (ICARCV), 2010 11th International Conference on IEEE, pp. 627-632 (2010). 
9. Hongxing S., Jiayi W., Peng S., and Zou X.: Facial Area Forecast and Occluded Face Detection Based on the YCbCr Elliptical Model. In Mechatronic Sciences, Electric Engineering and Computer (MEC), Proceedings 2013 international Conference on IEEE, pp. 1199-1202 (2013).

10. Zhang T., Li J., W. Jia, J. Sun and H. Yang: Fast and robust occluded face detection in ATM surveillance. Pattern Recognit. Lett., 107, 33-40 (2018).

11. Sikandar T., Ghazali K. H., and Rabbi M. F.: ATM Crime Detection using Image Processing Integrated Video Surveillance: A Systematic Review, Multi. Syst., 25(3), 229-251 (2019).

12. Kruegle H.: CCTV Surveillance: Video practices and technology. Elsevier (2011).

13. Centrix: Banking/Financial Institution. Centrixsecurity.com. http://www.centrixsecurity.com/markets/ban kingfinancial-institution/. Accessed 4 Jan 2017.

14. MegaPX ATM camera, 2016. https://www.anixter.com/content/dam/Suppliers/March\%20Networks/ Literature/MN_ATM-Camera_DS_EN_3-2016_LR.pdf. Accessed 10 September 2016.

15. Meech S.: Contemporary Quilts: Design, Surface and Stitch. Batsford (2003).

16. Prendergast P. M.: Facial Proportions. Adv. Surgical Facial Rejuvenation, Springer, Berlin, Heidelberg, 1522 (2012).

17. Bird B.M., Jofré V.S.C., Geniole S.N., Welker K.M., Zilioli S., Maestripieri D., Arnocky S. and Carre J.M.: Evolution and Human Behavior Does the facial width-to-height ratio map onto variability in men's testosterone concentrations ?. Evol. and Human Behav., 37(5), 392-39 (2016).

18. Mccormick C. M. and Carre J. M.: In your face : facial metrics predict aggressive behaviour in the laboratory and in varsity and professional hockey players. In Proc. of Royal Society London B: Biological Scienc., 275(1651), pp. 2651-2656 (2008).

19. Kovac J., Peter P. and Franc S.: Human skin color clustering for face detection. EUROCON 2003. Computer as a Tool. The IEEE Region 8, vol. 2, pp. 144-148 (2003).

20. Kakumanu P., Makrogiannis S., and Bourbakis N.: A survey of skin-color modeling and detection methods. Pattern Recog., 40(3), 1106-1122 (2007).

21. Tang Y., Zhang C., Gu R., Li P., and Yang B.: Vehicle detection and recognition for intelligent traffic surveillance system. Multi. Tools and Appl., 76(4), 5817-5832 (2017).

22. Black D. and Wilson J. C.: Internet video surveillance camera system and method. U.S.Patent No 7,659,922 (2010).

23. Honovich J. (2009): Security manager's guide to video surveillance. 3. IPVideoMarket. info.[online, ebook].

24. Velarde R. M. and Hung S. R.: Auto-triggered fast frame rate digital video recording. U.S. Patent No $8,830,339$ (2014).

25. Chen J. and Bai X.: Thermal face segmentation based on circular shortest path. Infrared Phys. Technol., 97, 391-400 (2019).

26. Knapik M. and Cyganek B.: Driver's fatigue recognition based on yawn detection in thermal images. Neurocomput,338, 274-292 (2019).

\section{Appendix A}

\section{Threshold value $\left(t_{1}\right)$ selection}

The algorithm uses facial area threshold value $\left(t_{l}\right)$ for full face cover classification. From the facial portion concept, facial region can be divided into equally spaced horizontal thirds. Therefore, a face is assumed as full 
$t_{1}$ value selection, facial area is determined using the total number of 1 (white) pixel in binary face image for the 90 bare face images of dataset in Table 1 . Then, the average facial area of the 90 bare face images is calculated and 1/3 of the average facial area is also calculated. It has been found that $1 / 3$ of the average facial area is 3384.65 . To further validate the reliability of the determined facial area threshold value $t_{1}$, predicted result in Receiver Operating Characteristic (ROC) space has been adopted. Different threshold values such as 1500, 1800, 2100, 2400, 2700, 3000, 3300, 3600, 3900 and etc are assumed randomly. The proposed method is applied using those threshold values on 180 full face cover images (90 robber's ski mask scenario and 90 niqab scenario). The corresponding correct detection rates and wrong detection rates are plotted in ROC space for the threshold values. The best predictive result of threshold value is considered as $t_{1}$. Table T1 shows the prediction result for different threshold value while Fig. A1 demonstrated the ROC space plot for $t_{1}$ selection. As demonstrated in Table T1 and Fig. A1 the threshold values less than $\mathrm{G}=3300$ may not give perfect result (correction rate $=1$ ) for any of the full face cover scenarios. The threshold value $G \geq 3300$ may produce perfect result (correction rate $=1$ ) for robber's ski mask, and nearly perfect result (correction rate $=0.97)$ for niqab. Therefore, the facial area threshold, $t_{1}$ for full face cover classification can be considered as $\mathrm{G}=3300$.

Table T1. Predicted Result for threshold value $t_{l}$ selection

\begin{tabular}{cccccc}
\hline Label no. & Threshold & \multicolumn{2}{c}{ Robber ski mask } & Niqab \\
\cline { 3 - 6 } & value & Correct detection & Wrong detection & Correct & Wrong \\
& & rate & rate & detection rate & detection rate \\
\hline A & 1500 & 0.18 & 0.82 & 0 & 1 \\
\hline B & 1800 & 0.47 & 0.53 & 0 & 1 \\
\hline C & 2100 & 0.67 & 0.33 & 0 & 0.67 \\
\hline D & 2400 & 0.73 & 0.27 & 0.33 & 0.17 \\
\hline E & 2700 & 0.84 & 0.16 & 0.87 & 0.13 \\
\hline F & 3000 & 0.89 & 0.11 & $\mathbf{0 . 9 7}$ & $\mathbf{0 . 0 3}$ \\
\hline G & $\mathbf{3 3 0 0}$ & $\mathbf{1}$ & $\mathbf{0}$ & 0.97 & 0.03 \\
\hline H & 3600 & 1 & 0 & 0.97 & 0.03 \\
\hline I & 3900 & 1 & 0 & & 0.03 \\
\hline
\end{tabular}

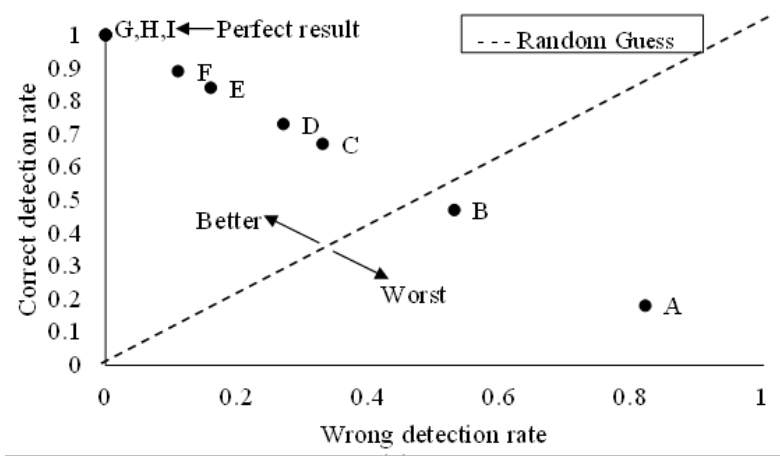

(a)

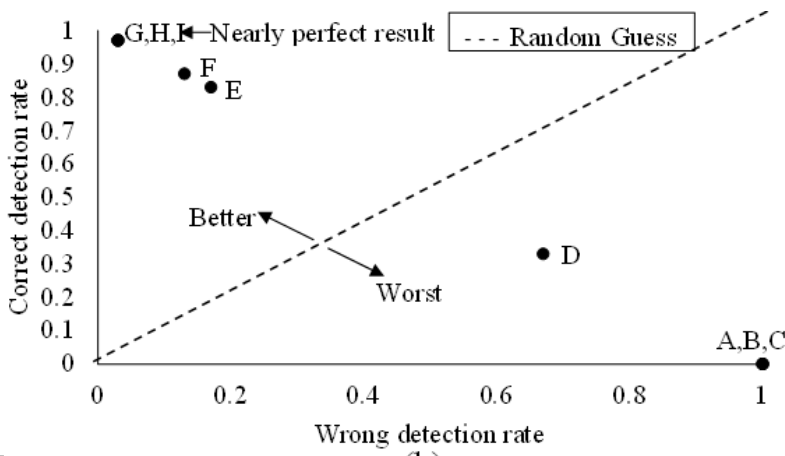

(b)

Fig. A1. ROC space plot of different threshold values for (a) robber's ski mask detection (b) niqab detection. 


\section{Appendix B}

\section{Threshold value $\left(t_{2}\right)$ selection}

The algorithm uses covered area percentage $\left(P_{c a}\right)$ threshold value $\left(t_{2}\right)$ for partially cover and uncover face classification. The experiment evaluates $P_{c a}$ value for the total 360 images of four facial scenarios including 90 bare face, 90 normal glass face, 90 sunglass face and 90 scarves face. An Average-Max-Min chart of covered area percentage value for the facial scenarios has been presented in Fig. B1. As demonstrated in the figure, the average $P_{c a}$ for bare face, normal glass face, sunglass face and scarves face are $2.32 \%, 4.47 \%, 16.53 \%$ and $49.71 \%$ respectively. From this result, it is evident that, average $P_{c a}$ for partially covered faces is much higher than that of uncovered faces. Moreover, the $P_{c a}$ value ranges from around $1 \%$ to $3 \%$ for bare face, $2 \%$ to $6.5 \%$ for normal glass faces, $10 \%$ to $18.5 \%$ for sunglass faces and $35 \%$ to $59 \%$ for scarves faces. This implies that, for uncover faces the $P_{c a}$ remains less than $6.5 \%$. On the other hand, the $P_{c a}$ of partially covered faces is greater than $10 \%$. Therefore, the best $P_{c a}$ threshold for partial covered face and uncovered face classification can be used as $t_{2}=10 \%$.

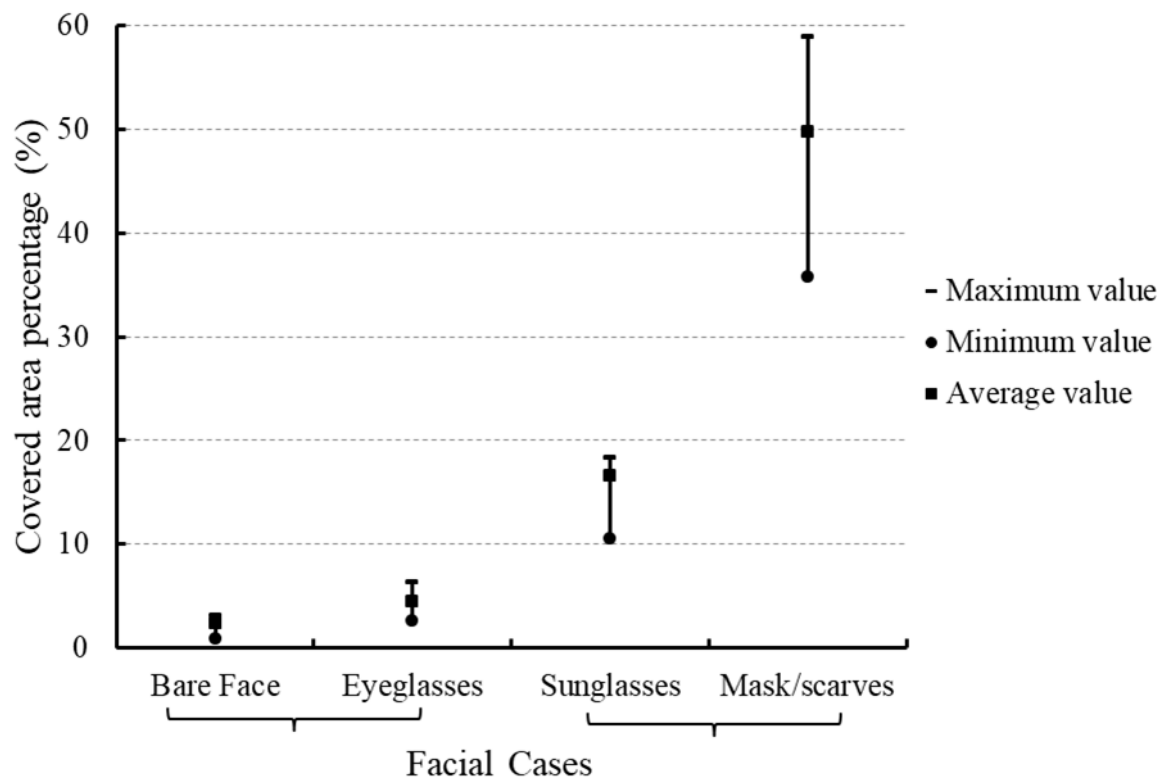

Fig. B1. Average-Max-Min chart plot of covered area percentage $\left(P_{c a}\right)$ value for all the images of uncover and partially cover facial scenarios. 